\title{
Two necropsy cases of chronic encephalomyelitis: variants of Neuro-Behcet's syndrome?
}

\author{
EIZO ISEKI, ${ }^{*}$ KIYOSHI IWABUCHI, $\dagger$ SABURO YAGISHITA, NAOJI AMANO, $\dagger$ \\ MASAAKI MATSUSHITA* \\ From the Department of Psychiatry and Neurology, Yokohama City University School of Medicine, ${ }^{*}$ the \\ Divisions of Psychiatry and Neurology, $\dagger$ and Pathology, $\ddagger$ Kanagawa Rehabilitation Center, Japan
}

SUMMARY Two necropsy cases of chronic encephalomyelitis of unknown aetiology are presented. Skin hyperreactivity occurred in both cases although there were no mucocutaneo-ocular symptoms. There was confluent perivenous necrosis with marked glio-mesenchymal reactions and persistent inflammatory changes, predominantly in the diencephalon and brain stem. These cases are clinicopathologically analogous to Neuro-Behcet's syndrome.

In 1957, Bickerstaff ${ }^{1}$ described cases of encephalitis in which there was almost total suppression of brain stem functions with complete recovery. He called them "brain stem encephalitis". In 1963, Iizuka ${ }^{2}$ reported three fatal cases of chronic brain stem encephalitis of unknown aetiology, and considered them to be in a specific category distinguished from other known encephalitides.

In this paper, we report two necropsy cases of chronic encephalomyelitis, resembling those reported by Iizuka. On the other hand, these cases are also quite similar to Neuro-Behcet's syndrome (NBS) except for the absence of mucocutaneo-ocular symptoms, suggesting that they may be variants of NBS.

\section{Case reports}

Case 1 was a male aged 36 years. In 1973, at the age of 26 years character changes such as childishness and aphrodisia were noticed. He temporarily developed claudication of the left leg in 1976, and double vision and dysarthria in 1980. In December 1981, gait disturbance and dysarthria rapidly progressed with double incontinence. The next month, on admission, his mentality was euphoric without appreciable dementia. Dysphagia and dysarthria were apparent. He showed increased deep tendon reflexes at the extremities with

\begin{abstract}
Address for reprint requests: Eizo Iseki, MD, Yokohama City University School of Medicine, 3-46 Urafune-cho, Minami-ku, Yokohama 232, Japan.
\end{abstract}

Received 3 November 1987 and in revised form 10 February 1988. Accepted 7 March 1988 positive Babinski's sign, slight diminution of superficial sensation in the lower limbs, and positive snout reflex. In July 1982, spastic paralysis appeared in the lower limbs. Thereafter he developed spastic paralysis of the right arm in October and tetraplegia in April 1983. In June, weakness of visual acuity and sensory impairment below the cervical level became apparent. The next month, he suddenly died of respiratory failure. During the clinical course there had been no evidence of uveitis, genito-oral ulcers or skin eruption, although phlebitis frequently occurred at the site of injections. On examination, CSF revealed pleocytosis between $15 \mathrm{~mm}^{3}$ and $150 \mathrm{~mm}^{3}$ as well as slightly increased total protein. EEG was almost normal until the terminal stage. CT showed dilatation of the lateral and third ventricles and progressive brain stem atrophy.

Necropsy findings The brain weighed $1,210 \mathrm{~g}$ and was atrophic, particularly in the brain stem. The spinal cord was also atrophic. The leptomeninges were slightly thickened. The ventricles were moderately dilated. There were no atheromatous plaques in the basal arteries. Microscopically the whole central nervous system was involved. In the cerebrum (fig a), the subcortical gray matter showed marked astrocytic proliferation and relatively mild neuronal loss with hyperplasia and hyperaemia of the capillaries and venules in the hypothalamus, subthalamic nucleus, ventral part of thalamus and globus pallidus. These changes were marked around small veins, frequently forming necrotic foci. In the white matter, there were confluent perivenous necrotic foci composed of destroyed myelin and axons, many lipid-laden macrophages and gliomesenchymal reactions in the cerebral peduncle, internal capsule, corpus callosum and optic nerves. Veins in these lesions often showed pronounced lymphocytic infiltration into the vessel wall and perivascular space (fig b), and the perivascular glial tissue was hyperplastic (fig c). Minute necrotic foci were scattered in the cerebral cortex, 

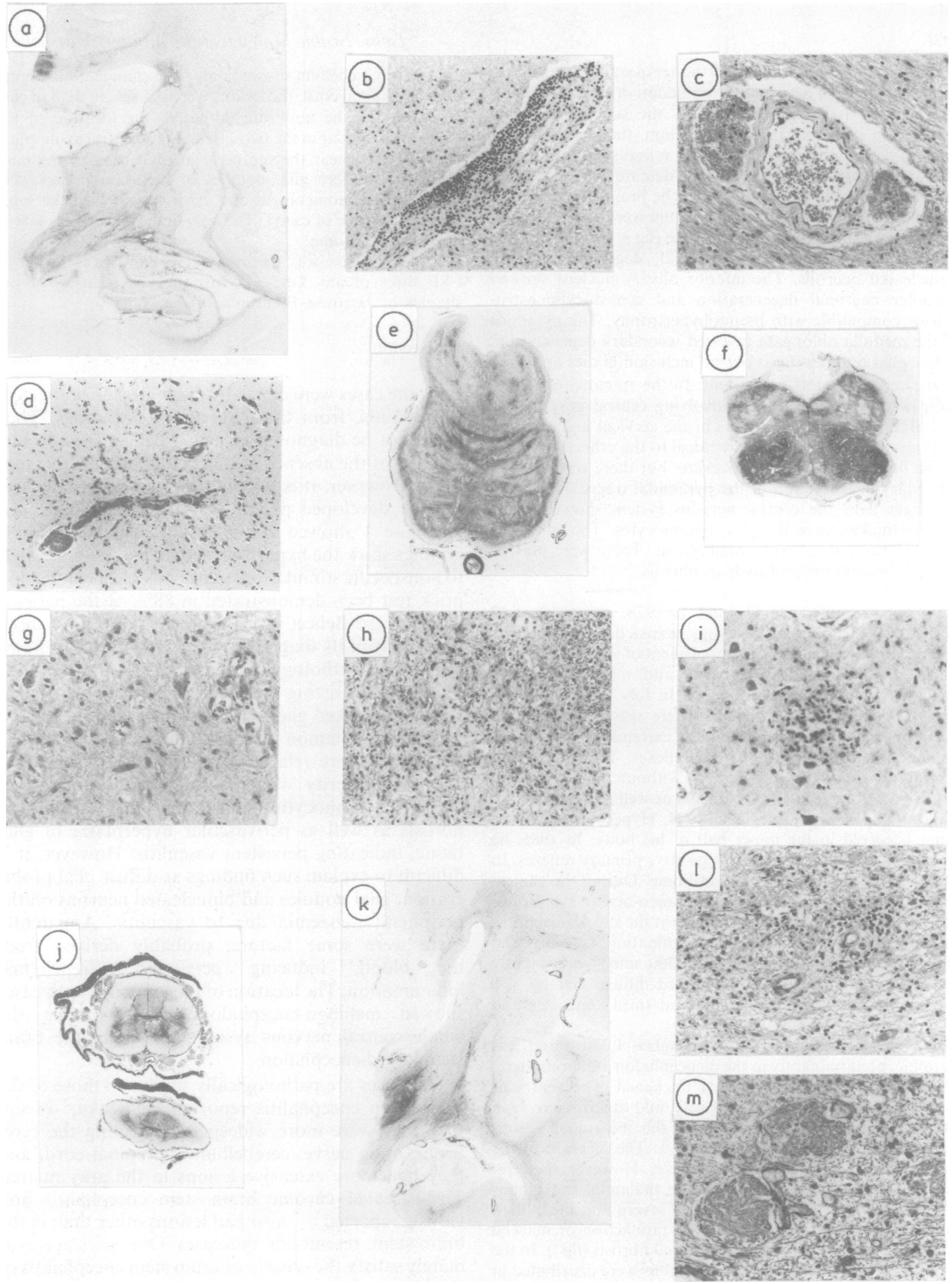

Figure (a) Gliosis in the midbrain, diencephalon and cerebral white matter. Case 1, Holzer. (b) Vascular lymphocytic infiltration in the globus pallidus. Case 1, HE $\times 90$. (c) Perivascular glial tissue in the pons. Case 1, HE $\times 100$. (d) Small perivenous necrosis in the cerebral cortex. Case $1, \mathrm{HE} \times 60 .(e, f)$ Marked gliosis in the pons and medulla oblongata. Case 1, Holzer. (g) Astrocytic proliferation and mild neuronal loss in the dorsal vagal nucleus. Case $1, \mathrm{HE} \times 110$. (h) Confluent necrosis with many lipid-laden macrophages in the pontine pyramidal tract. Case $1, L F B+H E \times 110$. (i) Glial nodule in the thalamus. Case 1, HE $\times 130$. (j) Gliosis in the central gray matter and columns of the spinal cord. Case 1, Holzer. ( $k$ ) Marked gliosis emphasised in the thalamus and globus pallidus. Case 2, Holzer. (l) Rarefactive lesion with venous adventitial fibrosis in the globus pallidus. Case $2, \mathrm{HE} \times 110$. (m) Central axonomas in the tegmentum of the midbrain. Case 2 , Bodian $\times 150$ 
especially in the deep layers of the temporal cortex (fig d). These consisted of perivenous rarefaction or glial scars. Similar necrotic foci also occurred in the subcortical white matter and striatum. In the cerebellum, there were loss of Purkinje cells with torpedo formation and perivenous rarefaction in the granule cell layer. The dentate nucleus showed severe neuronal loss and gliosis. In the brain stem (fig e, f), lesions similar to those of the cerebrum were widely distributed in both the tegmentum and base (fig $g, h$ ). The pontine nucleus showed marked neuronal degeneration with binucleated neurons. The inferior olivary nucleus showed vacuolar neuronal degeneration and gemistocytic astrocytosis compatible with pseudohypertrophy. The pyramids of the medulla oblongata revealed secondary degeneration. A few glial nodules (fig i) but no inclusion bodies appeared in the cerebrum and brain stem. In the spinal cord (fig j), there were extensive lesions involving central gray matter and almost all of the columns in the cervical and thoracic sections. These lesions were identical to the others. Changes in the lumbar cord were less severe, but there was obvious secondary degeneration of the pyramidal tracts. The leptomeninges over the central nervous system showed slight fibrous thickening with a few lymphocytes. The ventricles revealed marked subependymal gliosis. There were present bronchopneumonia and hydronephrosis.

Case 2 was a male aged 57 years. In 1978, at the age of 49 years, fever of unknown aetiology persisted, with headache and general fatigue. In 1980, impairment of visual acuity was noted. In 1981, gait disturbance and memory disorder appeared with double incontinence. In July 1982, on admission, dementia was prominent. There was bilateral optic atrophy. Deep tendon reflexes at the extremities were accentuated with positive pathological reflexes. Thereafter these symptoms gradually progressed without remission. He developed dysarthria and dysphagia as well as anisocoria in 1983 and spastic paraplegia in 1984. Hyperhidrosis sometimes occurred in the upper half of his body. In 1985, he developed akinetic mutism with positive primary reflexes. In March 1986, he suddenly died at home. During the clinical course he had showed no mucocutaneo-ocular symptoms, but he frequently developed phlebitis at the site of injections, with positive skin prick test. On examination, CSF revealed pleocytosis between $60 \mathrm{~mm}^{3}$ and $300 \mathrm{~mm}^{3}$ with slightly increased total protein. EEG showed diffuse slowing. CT showed dilatation of the lateral and third ventricles and progressive brain stem atrophy.

Necropsy findings The brain weighed 1,370 $\mathrm{g}$ and was atrophic, predominantly in the diencephalon and brain stem. The leptomeninges were slightly thickened. The ventricles were moderately dilated. There was mild atherosclerosis in the basal arteries. Microscopically the individual lesions were essentially the same as in case 1 . The location of the lesions was also similar in the two cases. However, the cerebral lesions (fig k), especially in the thalamus and globus pallidus, were more extensive, more severe and older than those of case 1 . They often showed rarefaction or marked fibrillary gliosis with venous adventitial fibrosis (fig l). In the cerebral white matter, perivenous lesions were distributed in the centrum semiovale and in the internal capsule and corpus callosum. Optic nerves were completely replaced by fibrillary gliosis, accompanied by vascular fibrosis and a few lympho- cytes. The cerebellum showed only little change. In the brain stem and spinal cord, the lesions were less severe than in case 1, except for the midbrain, although the location of the lesions was similar in the two cases. A few central axonomas ${ }^{3}$ were detected near the central tegmental tract of the midbrain (fig $\mathrm{m}$ ). No glial nodules or inclusion bodies were found. The leptomeninges and ventricles showed the same changes as those of case 1 . The only finding elsewhere was of bronchopneumonia.

In neither case was there any elevation of the serum or CSF titres of any kind of virus such as measles, herpes simplex or Japanese B encephalitis virus.

\section{Discussion}

Both the cases were clinically diagnosed as brain stem encephalitis, from the CSF and CT findings. They could not be diagnosed as neuro-Behcet's syndrome, because of the absence of mucocutaneo-ocular symptoms. However, it is noteworthy that both cases frequently developed phlebitis at the site of injections and case 1 showed positive skin prick test. These findings show the hyperreactivity of cutaneous vessels to nonspecific stimulation. Especially has positive skin prick test been demonstrated in $88 \%$ of the patients with neuro-Behcet's syndrome, ${ }^{4}$ and has been significant in its diagnosis.

The histopathology of the two cases was essentially the same, consisting of confluent perivenous necrotic foci with marked glio-mesenchymal reaction. In contrast to the common ischaemic changes, nerve cells in the lesions were relatively well preserved compared with the severity of glial reaction. Blood vessels showed lymphocytic infiltration and adventitial fibrosis as well as perivascular hyperplasia of glial tissue, indicating persistent vasculitis. However, it is difficult to explain such findings as diffuse glial proliferation, glial nodules and binucleated neurons on the peripheral ischaemia due to vasculitis. Apparently there were some factors, probably derived from the blood, inducing persistent parenchymal inflammation. The location of the lesions in both cases showed meningo-encephalomyelitis involving the whole central nervous system, especially the brain stem and diencephalon.

Our cases are pathologically similar to those of the brain stem encephalitis reported by Iizuka, ${ }^{2}$ except that they were more widespread affecting the cerebrum, optic nerve, cerebellum and spinal cord, and they had more extensive lesions in the gray matter. The cases of chronic brain stem encephalitis previously reported ${ }^{5-7}$ also had lesions other than in the brain stem, resembling our cases. Our cases approximately satisfy the criteria of brain stem encephalitis of lizuka type. ${ }^{8}$ It is not known whether the aetiology and pathogenesis are identical among these previous cases. Past investigators ${ }^{29}$ have compared them with 
viral encephalitides such as subacute sclerosing panencephalitis, herpes simplex and Japanese B encephalitis and demyelinating diseases such as multiple sclerosis and neuromyelitis optica. Our cases had no elevation of any viral titres in the serum or CSF, and showed no inclusion bodies directly suggesting viral infection. On the other hand, our cases can not be included in the demyelinating diseases, because the nature of the lesions was necrosis, not primary demyelination.

There were no stigmata nor pathological changes as collagen disease in the visceral organs and skin. The discrimination between our cases and Neuro-Behcet's syndrome, however, seems to be the most important. Neuro-Behcet's syndrome also has perivascular necrotic foci mainly involving the brain stem and shows vasculitis with lymphocytic cuffing. ${ }^{1011}$ Glial nodules and binucleated neurons observed in our cases are often found in Neuro-Behcet's syndrome as well as brain stem encephalitis of lizuka type. ${ }^{12}$ Pathologically it is difficult to distinguish our cases from Neuro-Behcet's syndrome so far as the CNS lesions are concerned. There have been many more reports of chronic brain stem encephalitis as well as Neuro-Behcet's syndrome in Japan ${ }^{13}$ than in other countries. ${ }^{14-16}$ Our cases may be reasonably considered as variants of Neuro-Behcet's syndrome, as Shiraki ${ }^{17}$ has suggested.

The fact that our cases showed the hyperreactivity of cutaneous vessels suggests that their pathogenesis is based on some allergic mechanism resembling NeuroBehcet's syndrome. It will be important in the future to examine in more detail the presence of vascular hyperreactivity in any cases of brain stem encephalitis and related disorders without mucocutaneo-ocular symptoms.

We thank Miss K Itoh and Mrs K Suzuki for their technical assistance.

\section{References}

1 Bickerstaff ER. Brain stem encephalitis. Further observations on a grave syndrome with benign prognosis. Br Med J 1957;1:1384-7.

2 Iizuka R. Uber Hirnstammencephalitis mit eigenartigem chronischem klinischen Verlauf. Acta Neuropathol (Berl) 1964:4:1-15.

3 Sung JH. Tangled masses of central axons (central axonomas) in the brain stem: Anatomical evidence for the regenerative growth of human central axons. $J$ Neuro-pathol Exp Neurol 1987:46:200-13.

4 Tüzün Y, Altac M, Yazici H, et al. Nonspecific skin hyperreactivity in Behcet's disease. Haematologica 1980;65:395-8.

5 Tatetsu S, Toya G, Miyakawa T. An autopsy case of brain stem encephalitis. Psychiatr Neurol Jap 1968;70:1-10.

6 Shicabe T, Tatsuta E, Kuroiwa Y, Tanaka K. An autopsy case of brain stem encephalitis with spinal cord involvement. Folia Psichiatr Neurol Jap 1972:26:133-43.

7 Ueno T, Takahata N. Chronic brainstem encephalitis with mental symptoms and ataxia. Report of three cases with necropsy. J Neurol Neurosurg Psychiatry. 1978;41:516-24.

8 lizuka R. Brain stem encephalitis. In: Nakazawa T, Yokoi S, eds. Modern Psychiatry Series, Vol 19C. Tokyo: Nakayama-shoten 1979:53-4 (in Japanese).

9 Shiraki H. Three autopsy cases of subacute to chronic disseminated encephalomyelitis. brain stem type. Neuropat Pol 1966;4 (Suppl):709-16.

10 Rubinstein LJ. Urich H. Meningo-encephalitis of Behcet's disease. Case report with pathological findings. Brain 1963;86:151-63.

11 Totsuka S, Hattori T, Yazaki M, Nagao K, Mizushima S. Clinicopathologic studies on Neuro-Behcet's disease. Folia Psychiatr Neurol Jap 1985;39:155-66.

12 Ikuta F, Kumanishi T. Oyanagi S. Koizumi T, Yamamura Y. Pathoetiological investigation of neuroBehcet disease. Adv Neurol Sci 1972:16:179-85 (in Japanese).

13 Ishino H. Brain stem encephalitis. In: Inose M. Ohtsuki S, Nakazawa T, eds. Modern Psychiatry Series, Vol 13A. Tokyo: Nakayama-shoten 1975:207-12 (in Japanese).

14 Verhaart WJC. A case of brain stem encephalitis. Neuropat Pol 1966:4 (Suppl):701-3.

15 Marsal J. Étude anatomo-clinique d'un cas déncéphalite du tronc cérébral. Rev Neurol (Paris) 1967:116: 141-60.

16 Waxman SG, Sabin TD, Embree LJ. Subacute brain stem encephalitis. J Neurol Neurosurg Psychiatry. 1974;37:811-16.

17 Shiraki H. Significance of neuropathology. In: Nakazawa T, Yokoi S, eds. Modern Psychiatry Series, Vol 19A. Tokyo: Nakayama-shoten 1978;54-60 (in Japanese). 